\title{
Intraoperative predictors of early tracheal extubation after living-donor liver transplantation
}

\author{
Serin Lee, Gye Jeol Sa, Stephanie Youna Kim, and Chul Soo Park
}

Department of Anesthesiology and Pain Medicine, Seoul St. Mary's Hospital, The Catholic University of Korea College of Medicine, Seoul, Korea

Background: Prolonged mechanical ventilation after liver transplantation has been associated with deleterious clinical outcomes, so early tracheal extubation posttransplant is now increasing. However, there is no universal clinical criterion for predicting early extubation in living-donor liver transplantation (LDLT). We investigated specific predictors of early extubation after LDLT.

Methods: Perioperative data of adult patients undergoing LDLT were reviewed. "Early" extubation was defined as tracheal extubation in the operating room or intensive care unit (ICU) within $1 \mathrm{~h}$ posttransplant, and we divided patients into early extubation $(\mathrm{EX})$ and non-EX groups. Potentially significant $(\mathrm{P}<0.10)$ perioperative variables from univariate analyses were entered into multivariate logistic regression analyses. Individual cut-offs of the predictors were calculated by area under the receiver operating characteristic curve (AUC) analysis.

Results: Of 107 patients, 66 (61.7\%) were extubated early after LDLT. Patients in the EX group showed shorter stays in the hospital and ICU and lower incidences of reoperation, infection, and vascular thrombosis. Preoperatively, model for end-stage liver disease score, lung disease, hepatic encephalopathy, ascites, and intraoperatively, surgical time, transfusion of packed red blood cell (PRBC), urine output, vasopressors, and last measured serum lactate were associated with early extubation $(\mathrm{P}<0.05)$. After multivariate analysis, only $\mathrm{PRBC}$ transfusion of $\leq 7.0$ units and last serum lactate of $\leq 8.2$ $\mathrm{mmol} / \mathrm{L}$ were selected as predictors of early extubation after LDLT (AUC 0.865).

Conclusions: Intraoperative serum lactate and blood transfusion were predictors of posttransplant early extubation. Aggressive efforts to ameliorate intraoperative circulatory issues would facilitate successful early extubation after LDLT. (Korean J Anesthesiol 2014; 67: 103-109)

Key Words: Living donors, Liver transplantation, Tracheal extubation.

Received: December 19, 2013. Revised: January 19, 2014. Accepted: February 7, 2014.

Corresponding author: Chul Soo Park, M.D., Ph.D., Department of Anesthesiology and Pain Medicine, Seoul St. Mary's Hospital, The Catholic University of Korea College of Medicine, 222, Banpo-daero, Seocho-gu, Seoul 137-701, Korea. Tel: 82-2-2258-2236, Fax: 82-2-537-1951, E-mail: p6c8s17@catholic.ac.kr

(c) This is an open-access article distributed under the terms of the Creative Commons Attribution Non-Commercial License (http:// creativecommons.org/licenses/by-nc/3.0/), which permits unrestricted non-commercial use, distribution, and reproduction in any medium, provided the original work is properly cited. 


\section{Introduction}

Prolonged mechanical ventilation after liver transplantation (LT) has been associated with many deleterious clinical outcomes. It can increase the risk of critical complications, such as pneumonia, sepsis, and multiorgan dysfunction [1]. Historically, mechanical ventilation with sedation or analgesia was advocated for up to $48 \mathrm{~h}$ posttransplant on the following theoretical bases: that it improved hemodynamic stability and facilitated early recovery of transplanted patients [2]. Recently, surgical techniques have improved sufficiently to shorten the overall surgical time and to avoid unexpected episodes of intraoperative bleeding. In particular, ultra-short-acting anesthetics have evolved to accelerate the LT recipient's recovery from anesthesia. Thus, early posttransplant extubation has gradually become established as a standard LT protocol without great objection from clinicians [3].

"Early" extubation in LT indicates immediate tracheal extubation in the operating room (OR) or intensive care unit (ICU) within $1 \mathrm{~h}$ posttransplant [4]. If a patient who undergoes early posttransplant extubation is transferred directly to a surgical ward without an ICU stay, the expression "fast tracking" may be used in place of early extubation. Early extubation is an essential component of fast tracking, and the patient undergoes major recovery in the post-anesthesia care unit $[5,6]$. Fast tracking was actively adopted in cardiac surgery during the 1980s [7], and Mandell et al. [8] first used this method with LT patients in 1997. In 2005, the incidence of early tracheal extubation had reached $60-80 \%$ of LT patients in Europe $[6,9]$.

However, determination of the appropriate tracheal extubation timing and conditions remains important in securing safe and reasonable patient recovery after LT. For these reasons, many clinical studies have been conducted in attempts to find predictors for early tracheal extubation. No definitive or universal criteria have yet been established regarding predictors for early tracheal extubation in LT patients.

In many Asian countries, living-donor liver transplantation (LDLT) is preferred over deceased-donor LT because of the shortages of deceased-donor donations [10]. LDLT has many different features in terms of surgical complexity and graft-related factors. Thus, the evaluation of conditions for early tracheal extubation in LDLT may differ considerably from that in deceaseddonor LT.

The aim of the present study was to identify factors for predicting early tracheal extubation in LDLT. Furthermore, we sought to assess the beneficial effects of early tracheal extubation in LDLT on posttransplant outcomes.

\section{Materials and Methods}

In total, 111 adult patients (of age $\geq 18$ years) who underwent
LDLT from January 2011 to December 2012 at our hospital were included in this retrospective study. Retransplantation cases were excluded. The Institutional Review Board of our hospital approved the study protocol and patient data collection. The electronic medical recording system and patient charts were used for data collection.

LDLT was performed with the right hepatic lobes of donors using a piggyback technique without veno-venous bypass. In each case, portal vein anastomosis was followed by hepatic artery anastomosis and bile duct reconstruction. A portocaval shunt was used in patients who had minimal collateral circulation, as evaluated from preoperative computed tomography, and a difference between the portal venous pressure and central venous pressure of more than $5 \mathrm{mmHg}$ after clamping the portal vein. All donor liver grafts were prepared with histidine-tryptophan-ketoglutarate solution.

Balanced anesthesia was conducted using inhaled anesthetic gas (desflurane, sevoflurane, or isoflurane) supported by an opioid (remifentanil or fentanyl) and a muscle relaxant (atracurium, vecuronium, or rocuronium) under a $40-50 \%$ oxygen/air mixture. Mechanical ventilation was controlled in the $\mathrm{PaCO}_{2}$ range of $30-35 \mathrm{mmHg}$ using a tidal volume of $8-10 \mathrm{ml} / \mathrm{kg}$ and a rate of 10-14 breaths/min. After induction of anesthesia, a SwanGanz catheter was placed through the right internal jugular vein for hemodynamic monitoring and cardiac output measurement, and the radial artery was cannulated using a 22-gauge angiocatheter for blood sampling and continuous monitoring of blood pressure. Intraoperative patient management was guided by the LDLT protocol of our hospital. Packed red blood cells (PRBCs) were administered to maintain the hematocrit between 25 and 30\%. Fresh frozen plasma, cryoprecipitate, and platelets were used to improve intraoperative coagulopathy under thromboelastography guidance. Vasopressors were administered when dangerous hemodynamic instability was indicated by invasive hemodynamic monitors. Calcium chloride was administered when serum calcium levels dropped to less than $80 \%$ of the normal lower limit. Sodium bicarbonate was indicated when the serum $\mathrm{pH}$ was lower than 7.15 under adequate minute ventilation.

"Early" tracheal extubation was defined as tracheal extubation in the OR or ICU within $1 \mathrm{~h}$ posttransplant. Tracheal extubations were performed following conventional guidelines. Patients with preoperative severe encephalopathy ( $\geq$ grade III), an intubated state, a severe hypoxic state $\left(\mathrm{PaO}_{2} / \mathrm{FiO}_{2}<150\right)$, and compromised cardiac function ( $\geq$ class III by New York Heart Association [NYHA] functional classification) were excluded from early tracheal extubation. Under conditions such as tidal volume $\geq$ 4-6 ml/kg, respiratory rate $\leq 30-35$ breaths $/ \mathrm{min}, \mathrm{PaO}_{2} \geq 60$ mmHg under $\mathrm{FiO}_{2} \leq 0.4$, afebrile (body temperature $\leq 38^{\circ} \mathrm{C}$ ), no significant acidosis, adequate mentation, and stable vital signs, early tracheal extubation was attempted [11]. According to early 
tracheal extubation, patients were grouped into the early extubation (EX) and nonEX groups. Preoperative, intraoperative, and postoperative variables of recipients and donor-related variables were compared between the groups using the chi-squared test or Student's t-test.

Preoperative variables considered were age, sex, body mass index (BMI), diabetes mellitus, hypertension, lung and heart disease, Child-Pugh-Turcotte (CPT) class, "model for end-stage liver disease" (MELD) score, cirrhosis-related complications (pleural effusion, hepatorenal syndrome, varix, ascites $\geq 1 \mathrm{~L}$, hepatic encephalopathy, hepatopulmonary syndrome), instrumentation (hemodialysis, endotracheal intubation), and laboratory findings, including arterial blood gas analysis (ABGA). Intraoperatively, surgical time, anesthetics (inhaled anesthetics, opioid, muscle relaxant), continuous renal replacement therapy, hourly urine output, vasopressors, body temperature, serum lactate and glucose, $\mathrm{PaO}_{2}$, fluid solutions, and $\mathrm{PRBC}$ transfusion were considered. The postoperative variables examined were death, length of stay at the hospital and in the ICU, mechanical ventilation duration, reintubation, infectious complications (surgical site, urinary, pulmonary, and blood), graft dysfunction, vascular thrombosis, and bleeding episodes. The donor-related variables considered were age, BMI, fatty change in the grafted liver, and graft-to-recipient weight ratio.

Potentially significant preoperative and intraoperative variables $(\mathrm{P}<0.10)$ after univariate intergroup comparisons were assessed by forward and backward stepwise multivariate logistic regression analyses. Data are presented as means \pm SDs or numbers (percentage) for results from the univariate analyses and odds ratio (95\% confidence interval, CI) for those from the multivariate analyses. The sensitivity and unbiased estimate of the logistic predicting model and the cutoff values of individual predictors from multivariate analysis were evaluated by area under the receiver operating characteristic curve (AUC) analysis. All tests were two-sided, and $\mathrm{P}$ values $<0.05$ were considered to indicate statistical significance. The SPSS software (ver. 15.0; SPSS Inc., Chicago, IL, USA) and MEDCALC (ver. 11.0 for Windows; MedCalc Software, Mariakerke, Belgium) were used for statistical analyses.

\section{Results}

In total, 107 subjects were included, having excluded 4 incomplete cases with missing data regarding the presence of early tracheal extubation or major perioperative parameters. The most common cause of liver disease was hepatitis B virus (71.0\%),

Table 1. Preoperative Recipient Characteristics

\begin{tabular}{|c|c|c|c|}
\hline Characteristic & $\begin{array}{l}\text { EX group } \\
(\mathrm{n}=66)\end{array}$ & $\begin{array}{l}\text { Non-EX group } \\
\quad(\mathrm{n}=41)\end{array}$ & $\mathrm{P}$ \\
\hline \multicolumn{4}{|l|}{ Recipient preoperative } \\
\hline Age (yr) & $52.5 \pm 8.2$ & $54.6 \pm 9.5$ & NS \\
\hline Body mass index $\left(\mathrm{kg} / \mathrm{m}^{2}\right)$ & $24.0 \pm 4.1$ & $25.5 \pm 4.4$ & NS \\
\hline $\operatorname{Sex}(M / F)$ & $53(80.3) / 13(19.7)$ & $28(68.3) / 13(31.7)$ & NS \\
\hline Cause of liver disease (HBV/HCV/alcohol/others) & $52(78.8) / 2(3.0) / 7(10.6) / 5(7.6)$ & $24(58.5) / 2(4.9) / 7(17.1) / 8(19.5)$ & NS \\
\hline Indication for liver transplantation (cirrhosis/cancer/combined) & $25(37.9) / 16(24.2) / 22(33.3)$ & $21(51.2) / 6(14.6) / 12(29.3)$ & NS \\
\hline Child-Pugh-Turcotte class (A and B/C) & $55(83.3) / 11(16.7)$ & $23(56.1) / 18(43.9)$ & 0.001 \\
\hline MELD score (points) & $12.1 \pm 6.5$ & $18.7 \pm 8.9$ & $<0.001$ \\
\hline Diabetes mellitus & $15(22.7)$ & $14(34.1)$ & NS \\
\hline Hypertension & $15(22.7)$ & $8(19.5)$ & NS \\
\hline Heart disease & $1(1.5)$ & $2(4.9)$ & NS \\
\hline Lung disease & $0(0)$ & $5(12.2)$ & 0.007 \\
\hline Pleural effusion & $3(4.5)$ & $4(9.8)$ & NS \\
\hline Hepatic encephalopathy ( $\geq$ grade II) & $9(13.6)$ & $16(39.0)$ & 0.003 \\
\hline Hepatorenal syndrome & $2(3.0)$ & $6(14.6)$ & NS \\
\hline Ascites $(\geq 1 \mathrm{~L})$ & $20(30.3)$ & $29(70.7)$ & $<0.001$ \\
\hline Hemodialysis & $2(3.0)$ & $5(12.2)$ & NS \\
\hline \multicolumn{4}{|l|}{ Laboratory findings } \\
\hline Hematocrit (\%) & $33.3 \pm 6.5$ & $27.9 \pm 4.6$ & $<0.001$ \\
\hline Platelet $\left(\times 10^{9} / \mathrm{L}\right)$ & $94.2 \pm 72.0$ & $62.8 \pm 42.1$ & 0.005 \\
\hline Sodium (mEq/L) & $139.2 \pm 4.0$ & $136.7 \pm 5.2$ & 0.006 \\
\hline Ammonia ( $\mu \mathrm{g} / \mathrm{dl})$ & $122.0 \pm 65.1$ & $133.7 \pm 85.9$ & NS \\
\hline Creatinine (mg/dl) & $0.88 \pm 0.47$ & $1.01 \pm 0.62$ & NS \\
\hline SGPT (U/L) & $59.7 \pm 182.0$ & $71.2 \pm 180.1$ & NS \\
\hline $\mathrm{pH}$ & $7.43 \pm 0.03$ & $7.43 \pm 0.04$ & NS \\
\hline $\mathrm{PaO}_{2}(\mathrm{mmHg})$ & $104.0 \pm 22.5$ & $103.1 \pm 29.9$ & NS \\
\hline
\end{tabular}

Data are presented as means \pm SD or numbers (\%). EX: early tracheal extubation, HBV: hepatitis B virus, HCV: hepatitis C virus, MELD: model for end-stage liver disease, SGPT: serum glutamic pyruvic transaminase, NS: not statistically significant $(\mathrm{P}>0.05)$. 
Table 2. Comparison of Recipient Intraoperative and Donor Variables

\begin{tabular}{|c|c|c|c|}
\hline & $\begin{array}{l}\text { EX group } \\
(\mathrm{n}=66)\end{array}$ & $\begin{array}{l}\text { Non-EX group } \\
\quad(\mathrm{n}=41)\end{array}$ & $\mathrm{P}$ \\
\hline \multicolumn{4}{|l|}{ Recipient Intraoperative Variable } \\
\hline Surgical time (min) & $446.0 \pm 63.7$ & $546.4 \pm 124.7$ & $<0.001$ \\
\hline Continuous renal replacement therapy & $1(1.5)$ & $3(7.3)$ & NS \\
\hline Inhaled anesthetics (desflurane/sevoflurane/isoflurane) & $24(38.7) / 23(37.1) / 15(24.2)$ & $11(28.2) / 21(53.8) / 7(17.9)$ & NS \\
\hline Use of remifentanil & $55(82.3)$ & $34(82.9)$ & NS \\
\hline Muscle relaxant infusion (atracurium/esmeron/vecuronium) & $61(92.4) / 1(1.5) / 4(6.1)$ & $36(87.8) / 4(9.8) / 1(2.4)$ & NS \\
\hline Vasopressor infusion & & & 0.001 \\
\hline None & $45(68.2)$ & $15(36.6)$ & \\
\hline Dopamine or dobutamine & $8(12.1)$ & $4(9.8)$ & \\
\hline Epinephrine or norepinephrine & $13(19.7)$ & $22(53.7)$ & \\
\hline Packed red blood cells (units) & $4.0 \pm 2.9$ & $13.8 \pm 8.8$ & $<0.001$ \\
\hline Hourly urine output (ml/kg/h) & $1.9 \pm 1.2$ & $1.1 \pm 1.0$ & $<0.001$ \\
\hline Body temperature $\left({ }^{\circ} \mathrm{C}\right.$, last measured $)$ & $36.71 \pm 0.34$ & $36.70 \pm 0.44$ & NS \\
\hline \multicolumn{4}{|l|}{ Serum lactate $(\mathrm{mmol} / \mathrm{L})$} \\
\hline Last measured & $5.47 \pm 2.64$ & $7.16 \pm 3.93$ & 0.018 \\
\hline Changed from initial level & $3.56 \pm 2.25$ & $4.57 \pm 3.73$ & NS \\
\hline Arterial pH (last measured) & $7.31 \pm 0.07$ & $7.29 \pm 0.08$ & NS \\
\hline Blood glucose (mg/dl, last measured) & $230.4 \pm 52.9$ & $226.5 \pm 48.1$ & NS \\
\hline \multicolumn{4}{|l|}{ Donor Variable } \\
\hline Age (yr) & $32.9 \pm 10.5$ & $34.3 \pm 11.6$ & NS \\
\hline Body mass index $\left(\mathrm{kg} / \mathrm{m}^{2}\right)$ & $23.0 \pm 2.7$ & $23.2 \pm 3.1$ & NS \\
\hline Fatty change of graft by biopsy (\%) & $5.6 \pm 6.6$ & $5.2 \pm 8.4$ & NS \\
\hline Graft-to-recipient weight ratio (\%) & $1.27 \pm 0.35$ & $1.18 \pm 0.33$ & NS \\
\hline
\end{tabular}

Data are presented as means \pm SD or numbers $(\%)$. EX: early tracheal extubation, NS: not statistically significant $(\mathrm{P}>0.05)$.

and liver cirrhosis was a more common indication for LDLT than hepatic cancer (43.0 vs. $20.6 \%$ ). In total, 66 patients $(61.7 \%)$ underwent early tracheal extubation after LT. Table 1 shows a comparison of preoperative characteristics between the EX and non-EX groups. Preoperative hepatic disease severity was milder in the EX group than the non-EX group. Patients in the EX group had lower MELD scores and incidences of CPT class C. The incidence of pulmonary disease and cirrhosis-related complications, such as hepatic encephalopathy and large ascites, was also lower in the EX group than in the non-EX group. However, no intergroup difference was found in ABGA findings, including $\mathrm{PaO}_{2}$ and $\mathrm{pH}$.

Total surgical time was shorter in the EX group than the nonEX group. Surgical time $8.5 \mathrm{~h}$ was a cut-off value for determination of posttransplant early tracheal extubation (AUC 0.774, sensitivity $84.1 \%$, specificity $61.0 \%, \mathrm{P}<0.001$ ). Intraoperatively, anesthetic agents showed no influence on the performance of early tracheal extubation. Patients in the EX group had lower $\mathrm{PRBC}$ transfusion volumes during surgery than patients in the non-EX group $(4.0 \pm 2.9$ vs. $13.8 \pm 8.8$ units, respectively; $\mathrm{P}<$ 0.001). Intraoperative circulatory status was better in the EX group, as indicated by lower serum lactate $(5.47 \pm 2.64$ vs. 7.16 $\pm 3.93 \mathrm{mmol} / \mathrm{L}$ in the non-EX group, $\mathrm{P}=0.018$ ) at the end of surgery, higher hourly urine output, and lower use of potent vasopressors. No donor factor assessed was associated with early tracheal extubation after LDLT (Table 2).
Table 3. Comparison of Posttransplant Outcomes according to Early Tracheal Extubation after Living-donor Liver Transplantation

\begin{tabular}{lccc}
\hline \multicolumn{1}{c}{ Characteristic } & $\begin{array}{c}\text { EX group } \\
(\mathrm{n}=66)\end{array}$ & $\begin{array}{c}\text { Non-EX group } \\
(\mathrm{n}=41)\end{array}$ & $\mathrm{P}$ \\
\hline ICU stay (d) & $5.7 \pm 1.6$ & $8.8 \pm 7.3$ & 0.011 \\
Hospital stay (d) & $24.4 \pm 14.8$ & $31.0 \pm 14.6$ & 0.026 \\
Death & $1(1.5)$ & $1(2.4)$ & $\mathrm{NS}$ \\
Reintubation & $1(1.5)$ & $4(9.8)$ & $\mathrm{NS}$ \\
Reoperation & $1(1.5)$ & $5(12.2)$ & 0.030 \\
Infectious complication & $5(7.6)$ & $9(22.0)$ & 0.032 \\
Graft dysfunction & $1(1.5)$ & $3(7.3)$ & $\mathrm{NS}$ \\
Vascular thrombosis & $0(0)$ & $2(4.9)$ & $<0.001$ \\
Bleeding episode & $1(1.5)$ & $4(9.8)$ & $\mathrm{NS}$ \\
\hline
\end{tabular}

Data are presented as means \pm SD or numbers (\%). EX: early tracheal extubation, ICU: intensive care unit, NS: not statistically significant $(\mathrm{P}>0.05)$.

After LDLT, patients in the EX group stayed in the hospital (24.4 vs. 31.0 days, $\mathrm{P}<0.05$ ) and ICU (5.7 vs. 8.8 days, $\mathrm{P}<0.05$ ) for shorter periods than those in the non-EX group. Although no intergroup difference was found in mortality, the incidences of reoperation, infectious complications, and vascular thrombosis were significantly lower in the EX group (Table 3 ).

The final predicting model for early tracheal extubation in LDLT included only two intraoperative factors: PRBC transfusion and last measured serum lactate (Table 4). AUC analysis indicated that this predictive model had clinically suitable ac- 
Table 4. Independent Predictors of Early Tracheal Extubation after Living-donor Liver Transplantation by Multivariate Logistic Regression

\begin{tabular}{lrrrrrrr}
\hline & $\beta$ & Odds ratio & $95 \% \mathrm{CI}$ & $\mathrm{P}$ & AUC & Sensitivity (\%) & Specificity (\%) \\
\hline Intraoperative & & & & & & & \\
PRBC transfusion $(\leq 7.0$ units) & 3.14 & 23.2 & $7.71-69.70$ & $<0.001$ & 0.902 & 86.4 & 78.0 \\
Lastly measured serum lactate $(\leq 8.2 \mathrm{mmol} / \mathrm{L})$ & 1.85 & 6.34 & $1.74-23.08$ & 0.005 & 0.614 & 89.4 & 41.5 \\
\hline
\end{tabular}

AUC of overall predicting model: $0.865(\mathrm{P}<0.001)$. AUC: area under receiver operating characteristic curve, PRBC: packed red blood cells.

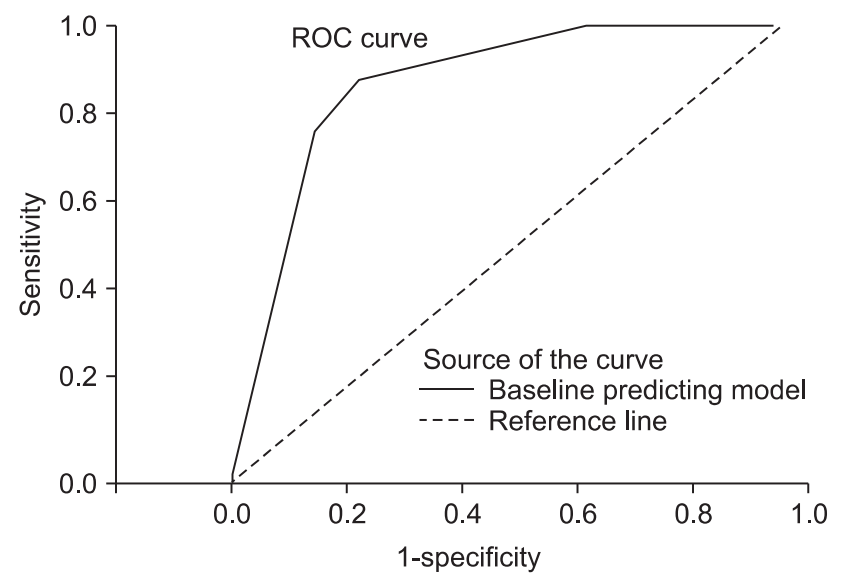

Fig. 1. ROC curve of the predictive model for early tracheal extubation after living-donor liver transplantation. ROC: receiver operating characteristic.

curacy for the prediction of early extubation (AUC 0.865, sensitivity/specificity $86.4 \% / 78.0 \% ; \mathrm{P}<0.001$ ) (Fig. 1). Individual cut-offs of predictors were PRBC transfusion $\leq 7.0$ units and last measured serum lactate $\leq 8.2 \mathrm{mmol} / \mathrm{L}$. Transfusion of PRBCs was the single factor with the greatest predictive potency for early tracheal extubation after LDLT (AUC 0.902, odds ratio 23.2).

\section{Discussion}

The rate of early tracheal extubation after LDLT in our center exceeded $60 \%$, approaching that seen in Western countries. Early tracheal extubation after LT has increased gradually over the last 20 years, and now has a place in standard LT protocols $[3,4,12]$. In the present study, more than three-quarters of LT patients were anesthetized with ultra short-acting anesthetics, such as desflurane, sevoflurane, remifentanil, and atracurium. Although the choice of anesthetic agents was not a key factor in determining early posttransplant tracheal extubation, the pharmacodynamic properties and clinical popularity of the current anesthetic agents are undeniable parameters in the background that permit early tracheal extubation in LT.

The importance of uncomplicated surgical progression was also emphasized by the contribution of shorter surgical times and lower blood transfusion volumes in patients with early tracheal extubation. Although LDLT is associated with compara- tively longer surgical durations than does deceased-donor LT because of the greater surgical complexity [13], a surgical duration longer than $8.5 \mathrm{~h}$ in LDLT seemed to be inappropriate for successful early tracheal extubation.

Traditionally, overnight mechanical ventilation had been routinely advocated after LT for patient safety and securing better circulatory conditions for the graft $[2,14]$. Moreover, some hazards from inappropriate tracheal extubation, such as respiratory insufficiency and subsequent urgent reintubation, may have prevented courageous attempts at early extubation after LT in the past. However, prolonged mechanical ventilation has also been persistently criticized in association with ventilator-associated pneumonia, muscle conditioning, tracheal injury, and, ultimately, mortality [15]. Thus, the need for early extubation had been naturally considered a future target in LT patient management. In the present study, patients in the non-extubation group were supported by overnight mechanical ventilation for more than $14 \mathrm{~h}$ (median $14.0 \mathrm{~h}$, mean $14.3 \mathrm{~h}$ ). Higher incidences of posttransplant complications and longer stays in the hospital or ICU in the non-extubation group sufficiently showed the deleterious effects of long mechanical ventilation in LT. Besides the well-known infection rate associated with intubation, lower incidences of reoperation and vascular thrombosis suggest a wide prognostic association of early tracheal extubation. Thus, overnight mechanical ventilation after LT should be prudently attempted in the presence of reasonable clinical indications.

Several clinical studies have investigated the factors that contribute to early tracheal extubation or fast tracking after LT $[4,5,8,16]$. Predictors of delayed mechanical ventilation have mainly been noted in association with the success of early tracheal extubation in LT, including emergent LT, hemodynamic instability [17], a high MELD score [9], poorly controlled encephalopathy, a high BMI [18], retransplantation, Child-Pugh class $\mathrm{C}$ disease, and a large intraoperative blood transfusion volume [6].

However, it is difficult to find any systematically organized criteria for early tracheal extubation that can be readily applied in the clinical setting. In fact, we considered the "Safe Operating Room Extubation after Liver transplantation" (SORELT) criteria from 2010 to be the only criteria for early extubation that can be used practically [19]. This includes two major criteria - PRBC transfusion $<7$ units and serum lactate $<3.4 \mathrm{mmol} / \mathrm{L}-$ and 
three minor criteria - patient status at transplant, duration of surgery $<5 \mathrm{~h}$, and vasoactive drug infusions (dopamine $\leq 5 \mu \mathrm{g}$ / $\mathrm{kg} / \mathrm{min}$ or norepinephrine $\leq 0.05 \mu \mathrm{g} / \mathrm{kg} / \mathrm{min}$ ). When a patient shows two major criteria, one major plus two minor criteria, or three minor criteria, immediate extubation in the OR is indicated.

The results of the present multivariate analysis were similar to the SORELT criteria, but the SORELT criteria were developed originally from mainly deceased-donor LT (>95\%), and mixed types of LT including pediatric LT, retransplantation, and combined liverkidney transplantation. In contrast, the population in the present study was homogeneous; the donation type was limited to solely living donors, and pediatric and retransplantation cases were excluded.

The main components of our predictive model for early tracheal extubation were identical to the major components in the SORELT criteria, but our threshold for serum lactate was considerably higher than that in SORELT ( 8.2 vs. $3.4 \mathrm{mmol} / \mathrm{L}$ ). This increased value of serum lactate value may be due to some intrinsic property of LDLT. LDLT in our center uses the right hepatic lobe from the donor, which is nearly half the size of a deceased-donor liver. Thus, there may be insufficiency in clearing circulating lactate through the unrecovered liver graft. Also, the comparatively long surgical duration associated with LDLT could be another cause of accumulated serum lactate in the body.

Preoperative disease severity was noted to have a relationship with early extubation in univariate analyses. For example, MELD score and CPT class showed statistically significant associations with early tracheal extubation. A MELD score of $<11$ points was reportedly associated with rapid extubation in a previous study [9]. However, after multivariate adjustment with intraoperative factors in the present study, the significance of preoperative indicators in early tracheal extubation disappeared. In the SORELT criteria, most early extubation predictors were also intraoperative predictors. The preoperative admission status was the only preoperative predictor and was and classified as a minor component. The reason for the absence of preoperative factors is unclear, but might be associated with the influence of intraoperative factors in the multivariate analysis.

Indeed, only intraoperative factors formed the predicting model for early tracheal extubation after LDLT. A small volume of transfused blood may reflect few episodes of bleeding and an uncomplicated surgical progression during the surgery. The tight relationship between intraoperative blood transfusion with tracheal extubation has been shown in many studies in several clinical areas $[17,20,21]$. The cut-off value for PRBC transfusion (7 units) for early extubation in this study regarding LDLT was not a new finding and was largely consistent with one of the SORELT criteria. Serum lactate is a well-known clinical indicator of circulatory insufficiency and poor prognosis [22,23]. In the present study, low hourly urine output and administration of more potent vasopressors in the non-extubation group could indicate intraoperative circulatory insufficiency, subsequently connected with increasing serum lactate.

In LT, serum lactate acts as a prognostic indicator of hepatic failure and ultimately mortality [24,25]. In particular, the change in intraoperative serum lactate after hepatic allograft reperfusion can serve as a predictor of initial graft function in LDLT [26]. Accordingly, reduction in serum lactate at the end of surgery in this study could be interpreted as successful settlement of the grafted liver, which then begins to take up and metabolize serum lactate. Although donor factors did not affect early tracheal extubation, graft status and ultimate success in the surgical procedure could be evaluated indirectly through serum lactate levels.

The present study has some limitations. First, the absence of some data could not be avoided because of the retrospective study design. Although we attempted to cover the major factors affecting tracheal extubation, not all predictors of tracheal extubation could be encompassed because of the excessive number of potential variables. Second, the judgments about tracheal extubation were subjective, to some degree, and not uniform. Despite our efforts to adhere to the general guidelines for tracheal extubation, patient respiratory parameters could not always be fully assessed because of delirious states due to effects of residual anesthetic at the end of surgery. Third, this study did not include enough patients because our hospital does not handle a sufficient number of LT cases, and the rate of early tracheal extubation has increased markedly over the last 2 years.

Despite these limitations, the present study also has several strengths. First, previous known major predictors of early tracheal extubation in LT were simplified, and their applicability to LDLT was newly demonstrated here. The serum lactate threshold for early tracheal extubation was adjusted through this study, reflecting the different properties of LDLT. Second, the importance of intraoperative factors in early extubation after LDLT was emphasized, versus preoperative conditions.

In conclusion, the last measured intraoperative serum lactate level and blood transfusion were predictors of early tracheal extubation after LDLT. More aggressive efforts to ameliorate intraoperative circulatory issues by optimizing blood transfusion and serum lactate levels would facilitate successful early tracheal extubation after LDLT. 


\section{References}

1. Mueller AR, Platz KP, Kremer B. Early postoperative complications following liver transplantation. Best Pract Res Clin Gastroenterol 2004; 18: 881-900.

2. Plevak DJ, Southorn PA, Narr BJ, Peters SG. Intensive-care unit experience in the Mayo liver transplantation program: the first 100 cases. Mayo Clin Proc 1989; 64: 433-45.

3. Wu J, Rastogi V, Zheng SS. Clinical practice of early extubation after liver transplantation. Hepatobiliary Pancreat Dis Int 2012; 11: 577-85.

4. Mandell MS, Stoner TJ, Barnett R, Shaked A, Bellamy M, Biancofiore G, et al. A multicenter evaluation of safety of early extubation in liver transplant recipients. Liver Transpl 2007; 13: 1557-63.

5. Taner CB, Willingham DL, Bulatao IG, Shine TS, Peiris P, Torp KD, et al. Is a mandatory intensive care unit stay needed after liver transplantation? Feasibility of fast-tracking to the surgical ward after liver transplantation. Liver Transpl 2012; 18: 361-9.

6. Glanemann M, Busch T, Neuhaus P, Kaisers U. Fast tracking in liver transplantation. Immediate postoperative tracheal extubation: feasibility and clinical impact. Swiss Med Wkly 2007; 137: 187-91.

7. Quasha AL, Loeber N, Feeley TW, Ullyot DJ, Roizen MF. Postoperative respiratory care: a controlled trial of early and late extubation following coronary-artery bypass grafting. Anesthesiology 1980; 52: 135-41.

8. Mandell MS, Lockrem J, Kelley SD. Immediate tracheal extubation after liver transplantation: experience of two transplant centers. Anesth Analg 1997; 84: 249-53.

9. Biancofiore G, Bindi ML, Romanelli AM, Boldrini A, Bisa M, Esposito M, et al. Fast track in liver transplantation: 5 years' experience. Eur J Anaesthesiol 2005; 22: 584-90.

10. Chen CL, Kabiling CS, Concejero AM. Why does living donor liver transplantation flourish in Asia? Nat Rev Gastroenterol Hepatol 2013; 10: 746-51.

11. MacIntyre NR, Cook DJ, Ely EW Jr, Epstein SK, Fink JB, Heffner JE, et al. Evidence-based guidelines for weaning and discontinuing ventilatory support: a collective task force facilitated by the American College of Chest Physicians; the American Association for Respiratory Care; and the American College of Critical Care Medicine. Chest 2001; 120(6 Suppl): S375-95.

12. Ozier Y, Klinck JR. Anesthetic management of hepatic transplantation. Curr Opin Anaesthesiol 2008; 21: 391-400.

13. Niemann CU, Hirose R, Stock P, Roberts JP, Mandell S, Spencer Yost C. Intraoperative fluid management of living donor versus cadaveric liver transplant recipients. Transplant Proc 2004; 36: 1466-8.

14. Glanemann M, Langrehr J, Kaisers U, Schenk R, Müller A, Stange B, et al. Postoperative tracheal extubation after orthotopic liver transplantation. Acta Anaesthesiol Scand 2001; 45: 333-9.

15. Razonable RR, Findlay JY, O'Riordan A, Burroughs SG, Ghobrial RM, Agarwal B, et al. Critical care issues in patients after liver transplantation. Liver Transpl 2011; 17: 511-27.

16. Schultz NA, Larsen PN, Klarskov B, Plum LM, Frederiksen HJ, Christensen BM, et al. Evaluation of a fast-track program for patients undergoing liver resection. Br J Surg 2013; 100: 138-43.

17. Zeyneloglu P, Pirat A, Guner M, Torgay A, Karakayali H, Arslan G. Predictors of immediate tracheal extubation in the operating room after liver transplantation. Transplant Proc 2007; 39: 1187-9.

18. Mandell MS, Lezotte D, Kam I, Zamudio S. Reduced use of intensive care after liver transplantation: patient attributes that determine early transfer to surgical wards. Liver Transpl 2002; 8: 682-7.

19. Skurzak S, Stratta C, Schellino MM, Fop F, Andruetto P, Gallo M, et al. Extubation score in the operating room after liver transplantation. Acta Anaesthesiol Scand 2010; 54: 970-8.

20. Kipps AK, Wypij D, Thiagarajan RR, Bacha EA, Newburger JW. Blood transfusion is associated with prolonged duration of mechanical ventilation in infants undergoing reparative cardiac surgery. Pediatr Crit Care Med 2011; 12: 52-6.

21. Cywinski JB, Xu M, Sessler DI, Mason D, Koch CG. Predictors of prolonged postoperative endotracheal intubation in patients undergoing thoracotomy for lung resection. J Cardiothorac Vasc Anesth 2009; 23: 766-9.

22. Wenkui Y, Ning L, Jianfeng G, Weiqin L, Shaoqiu T, Zhihui T, et al. Restricted peri-operative fluid administration adjusted by serum lactate level improved outcome after major elective surgery for gastrointestinal malignancy. Surgery 2010; 147: 542-52.

23. Basaran M, Sever K, Kafali E, Ugurlucan M, Sayin OA, Tansel T, et al. Serum lactate level has prognostic significance after pediatric cardiac surgery. J Cardiothorac Vasc Anesth 2006; 20: 43-7.

24. Du WB, Pan XP, Li LJ. Prognostic models for acute liver failure. Hepatobiliary Pancreat Dis Int 2010; 9: 122-8.

25. Macquillan GC, Seyam MS, Nightingale P, Neuberger JM, Murphy N. Blood lactate but not serum phosphate levels can predict patient outcome in fulminant hepatic failure. Liver Transpl 2005; 11: 1073-9.

26. Nishimura A, Hakamada K, Narumi S, Totsuka E, Toyoki Y, Ishizawa Y, et al. Intraoperative blood lactate level as an early predictor of initial graft function in human living donor liver transplantation. Transplant Proc 2004; 36: 2246-8. 